\title{
LONGITUDINAL AND TRANSVERSE WAKEFIELD ANALYSIS IN A MULTI-MODE CYLINDRICAL STRUCTURE
}

\author{
Liling Xiao, Wei Gai and J. G. Power \\ Argonne National Laboratory, Argonne, IL 60439, USA
}

\begin{abstract}
We give a summary and a analysis of the wakefields in the dielectric channels. We found that using conventional description of the electromagnetic wave propagation in dielectric channel, we can derive the power flow and wakefield generations. Detailed analysis of the higher order HEM11 mode is also given including separation of TM and TE dominated modes. Numerical calculations of the longitudinal and transverse wakefileds in multimode structures are given.
\end{abstract}

\section{INTRODUCTION}

Wakefield generation in a dielectric channel (shown in Fig.1) has been investigated thoroughly in the past [1-2]. Recently, Parks et al calculated the wakefields [3] using normal mode expansion method and arrived the same wakefield expression as in [1-2]. In [1] and [2], a Green function was solved easily using the boundary condition. This method gives wakefield amplitude and dispersion relations at the same time. The dispersion relations were obtained easily using the poles in the contour integral while the amplitude was derived by simple integral over the poles.

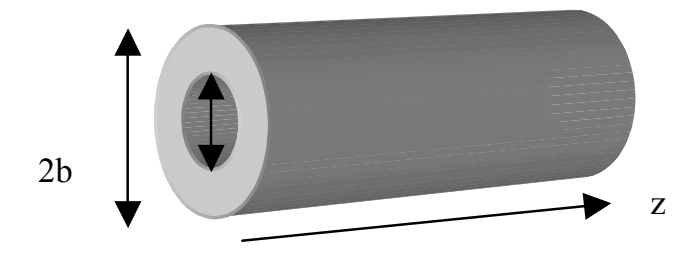

Figure 1 Dielectric lined waveguide as wakefield acceleration structure.

There were excitements about the newly proposed multi drive beam excited wakefield in a multimode structure $[3,4]$. Because the scheme uses large amount of excited modes for coherent wave construction, thus a very narrow peak of acceleration field can be obtained. The transverse wakefield would also be excited and constructed coherently. If the excitation frequencies spacing of very high frequency mode for monopole and dipole modes are different, then the transverse beam instability can easily be controlled. However, our calculation showed that unfortunately that all the higher order modes have the same frequency spacing thus constructed coherently at the same location, which is in agreement with [3]. Both our analytical approach and numerical calculation are given in this paper.

\section{WAVES AND WAKEFIELDS IN CYLINDRICAL DIELECTRIC CHANNELS}

The waves derived from Maxwell wave equation in a dielectric channel can be expressed and expanded as [5]

$$
\begin{aligned}
& \left(\begin{array}{l}
\mathbf{E}(r, z, \theta, t) \\
\mathbf{H}(r, z, \theta, t)
\end{array}\right)=\sum_{n=0}^{\infty} \sum_{m=0}^{\infty}\left(\begin{array}{c}
a_{m n} \mathbf{e}_{\mathbf{m n}}(r) \\
b_{m n} \mathbf{h}_{m n}(r)
\end{array}\right) e^{i m \theta} e^{i\left(k_{z} z^{-}-\omega t\right)}(1) \\
& \int \mathbf{e}_{\mathbf{m n}}(r) e^{i m \theta} \times \mathbf{h}_{\mathbf{m}^{\prime} \mathbf{n}^{\prime}}(r) e^{i m \cdot \theta} \bullet \vec{z} d s=\delta_{m m}, \boldsymbol{\delta}_{n n^{\prime}},(2)
\end{aligned}
$$

In general, $\mathbf{e}_{\mathrm{mn}}$ and $\mathbf{h}_{\mathrm{mn}}$ are the normal modes which are combinations of Bessel functions in cylindrical systems and they give a complete description of the wave properties in the system, including wakefields. The amplitude $a_{m n}$ and $b_{m n}$ depend on the excitations. Dispersion relation can be obtained by matching all the needed boundary conditions. For a highly relativistic beam, the longitudinal wakefields obtained in [1-3] can be expressed as

$$
\begin{aligned}
& \mathbf{E}_{\mathbf{z . m n}}(r)=\left\{\begin{array}{cc}
\eta_{m} I_{m}\left(k r_{0}\right) I_{m}(k r), & 0 \leq r \leq a \\
A_{m}\left[J_{m}(s a) Y_{m}(s r)-Y_{m}(s a) J_{m}(s r)\right], & a \leq r \leq b
\end{array}\right. \\
& \mathbf{H}_{\mathbf{z . m n}}(r)=\left\{\begin{array}{cc}
B_{m} I_{m}\left(k r_{0}\right) I_{m}(k r), & 0 \leq r \leq a \\
C_{m}\left[J_{m}^{\prime}(s a) Y_{m}(s r)-Y_{m}^{\prime}(s a) J_{m}(s r)\right], & a \leq r \leq b
\end{array}\right.
\end{aligned}
$$

All the other components can be derived from Equation (3). Where $\eta_{m}$ is excitation wakefield amplitude by a single charged particle. Then we can find the transverse wakefield using Panofsky and Wenzel theorem,

$$
\begin{aligned}
F_{r, m} & =\frac{e v}{j \omega} \frac{\partial E_{z, m}}{\partial r} \\
F_{\theta, m} & =\frac{e m v}{j \omega r} E_{z, m}
\end{aligned}
$$

For each individual mode $l$, due to the fact of the modes are orthogonal to each other, the energy loss per unit length for a single point charged particle e can be expressed in terms of the individual mode $l$, and the total energy loss is the sum of all the $W_{l}$.

$$
W_{l}=-\frac{1}{2} e \mathbf{E}_{z, l} \bullet \vec{z}
$$


Then the stored energy for each individual mode behind the drive beam is

$U_{l}=\int_{V}\left(\mathbf{E}_{1} \bullet \mathbf{E}_{1} *+\mathbf{H}_{1} \cdot \mathbf{H}_{1} *\right) d V$

As shown in [5], the power flow is

$P_{l}=\frac{1}{2} \int_{s}\left(\mathbf{E}_{1} \times \mathbf{H}_{1} *\right) \cdot d \vec{S}=v_{l, g} U_{l}$

Using energy conservation law, we have

$W_{l}=U_{l}\left(1-\frac{v_{l, g}}{c}\right)$

The above four equations completely describe the energy balance and energy flow in the dielectric channel. Using electron beam as a reference, then it means that the beam is radiating power into the structure and leaves the energy behind. But in the lab frame, the structure gained energy from the e-beam. This is because the energy is a scalar, thus the sign depends on the reference point as long as we have been consistent. However the direction of this energy flow is described by Poynting vector and it propagates in the same direction as the beam as in the form of Cerenkov radiation process, but at lesser speed of the beam.

\section{LONGITUDINAL AND TRANSVERSE MULTIMODE WAKEFIELD ACCELERATOR}

It was proposed in [4] that a multi-mode wakefield accelerator would enhance some physical properties such as coherence construction of the longitudinal modes would yield a much shorter RF packet, thus may lead to a high RF breakdown. It may also require a less intense single bunch beam. There were some ideas that the transverse mode separation would be different from fundamental longitudinal. However, both asymptotic approximate expansion and computer calculation showed that the mode spacing at high frequency excitations became equal for all the modes.

\subsection{Asymptotic expansion}

When a is very small, the wakefields of the TM branch in equations (1) can be approximated as

$$
E_{z}{ }^{m}=\eta \sum_{n} A_{m, n} J_{m}\left(s_{m, n} r\right) e^{i m \theta}
$$

where $s_{m n}$ is simply the root of Bessel function.

when $s_{m n}$ is very large, we can use the asymptotic expansion,

$$
J_{m}(x) \cong \sqrt{\frac{2}{\pi x}} \cos \left(x-\frac{\pi}{2} m-\frac{\pi}{4}\right)
$$

Then the root spacing $s_{m, n+1}-s_{m, n}$ is $\pi$, which is independent of $\mathrm{m}$ and $\mathrm{n}$. The frequency spacing can be calculated as following:

$$
\begin{aligned}
& k_{c, m, n}=\frac{s_{m, n}}{b}, f_{c, m n}=\frac{k_{c, m n} \times c}{2 \times \pi}, \\
& d f_{c}=\frac{c \times d k_{c, m n}}{2 \times \pi}=\frac{c}{2 b}, \\
& d f=\frac{d f_{c, m, n}}{\sqrt{\varepsilon \beta^{2}-1}}=\frac{c}{2 b \sqrt{\varepsilon \beta^{2}-1}}
\end{aligned}
$$

where $\beta$ is particle velocity and thus phase velocity of the wakefield.

The same results can also be obtained in TE branch using Hz. Thus the results show that all the higher order modes would not de-coherent from each other, thus the multimode structure does not lead to beam stability as we thought originally. Although the above treatment is for no-vacuum hole situation, numerical calculation indicates that the same conclusion applies to all the dielectric loaded structures as pointed out by Parks and Hirshfield [3], $\mathrm{df}=\mathrm{c} / 2(\mathrm{~b}-\mathrm{a})\left(\varepsilon \beta^{2}-1\right)^{1 / 2}$. In the next section, we give some numerical examples to illustrate properties of the transverse wakes.

\subsection{Numerical calculations}

We first consider a case using Alumina $(\varepsilon=9.43)$, with the inner radius $a=0.375 \mathrm{~mm}$, and outer radius $\mathrm{b}=4.38 \mathrm{~mm}$. This is the same as in reference[3]. Figure 2(a) shows the mode frequency separation approaches to the same value $(11.47 \mathrm{GHz})$ when the mode number exceeds 20 . The bunch length oz used here is $1 \mathrm{~mm}$. Figure 2(b) shows the normalized wakefield amplitudes for the dipole modes. This result shows clearly that TM mode dominate the transverse wakefield, and the transverse wakefield becomes coherent as the same way as the longitudinal wakefield as shown in Figure 2(c) and (d).

We have also calculated a dielectric structure with $\varepsilon=38.1, a=5 \mathrm{~mm}, b=14.4 \mathrm{~mm}$. The parameters used in this case calculation are the experimental setup performed at the AWA last year [4]. The bunch length $\sigma \mathrm{z}$ used here is $1 \mathrm{~mm}$. It is interesting that the Figure 3(a) shows the same trend as Figure 2(a). But, the wakefield amplitudes are dominated by the TE branch at the high frequency ranges as in Figure 3(b). This leads a relative de-coherent of transverse wakefield as shown in Figure 3(d). The longitudinal wakefield is shown in Figure 3(c), it shows very good coherent properties. In our experiment [4], we did not observe any effect of transverse wakefields.

\section{SUMMARY}

In this paper, we have clarified the concept of the power flow, stored energy and radiated energy into the dielectric from a beam. We also calculated the longitudinal and transverse wakefields in dielectriclined structures. We confirmed that all the higher order mode spacing goes to constant, therefore higher order transverse mode damping is necessary to implement so the beam stability can be obtained. The transverse 
modes can be dampened easily in the dielectric-lined structure by simply opening a few longitudinal slots with RF absorber materials placed outside [6]. Experiments and analytical analysis had shown that the transverse wake diminishes after a short distance. Since this scheme uses a pulse train instead of a very high charge single drive beam for wakefield generation. This dampen scheme is ideal for this scheme.

This work is supported by DOE, HEP Division under the contract No. W-31-109-ENG-38.
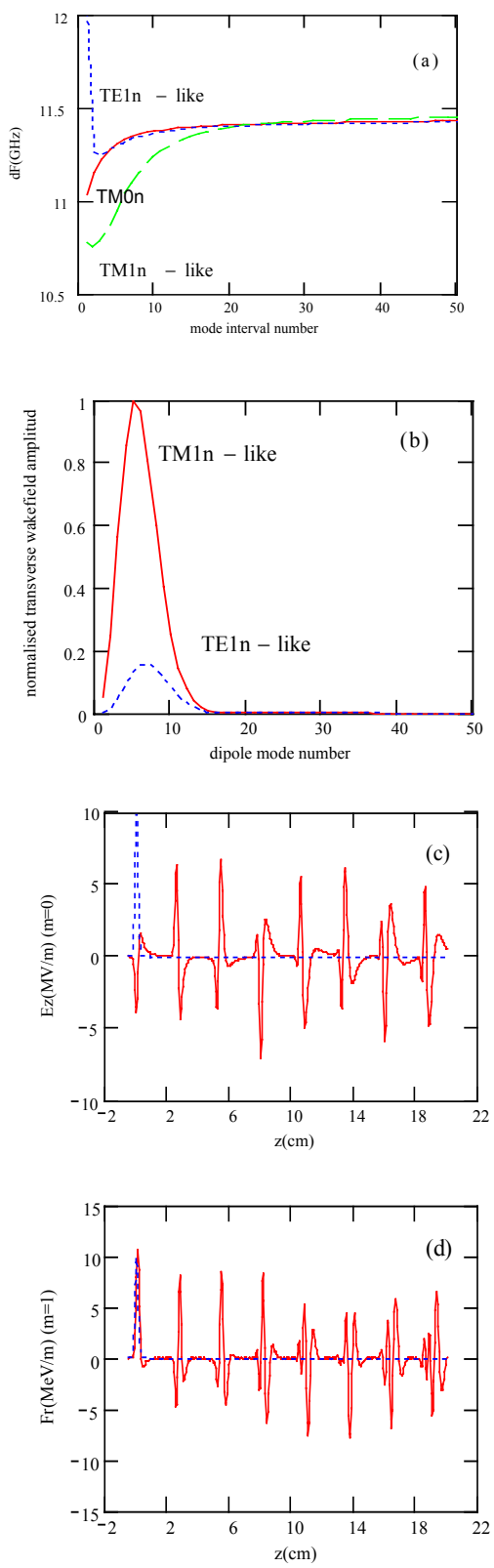

Fig.2 Calculated dielectric wakefield properties for TM dominated mode $(\mathrm{a}=0.375 \mathrm{~mm}, \mathrm{~b}=4.38 \mathrm{~mm}$, $\varepsilon=9.43, \sigma \mathrm{Z}=1 \mathrm{~mm}, \mathrm{Q}=1 \mathrm{nc}$ ) (a) frequency separation between adjacent modes, (b) normalized transverse wakefield amplitude, (c) longitudinal wakefield from a single bunch, (d) transverse force from a single bunch

\section{REFERENCE}

[1] W.Gai et al., Phys. Rev. Lett.24, 2756 (1988)

[2].K.Y.Ng, Phys. Rev. D42, 1819 (1990)

[3] S.Y.Park and J.L.Hirshfield, Phys. Rev. E62, 1266 (2000)

[4] J.G.Power et al., Phys.Rev.STAB, 101302 (2000)

[5] J.D.Jackson, Classical Electrodynamics, 2d ed.

(Wiley, New York, 1974)

[6] W.Gai and Ching-Hung Ho, J.Appl.Phys. 70(7) 395
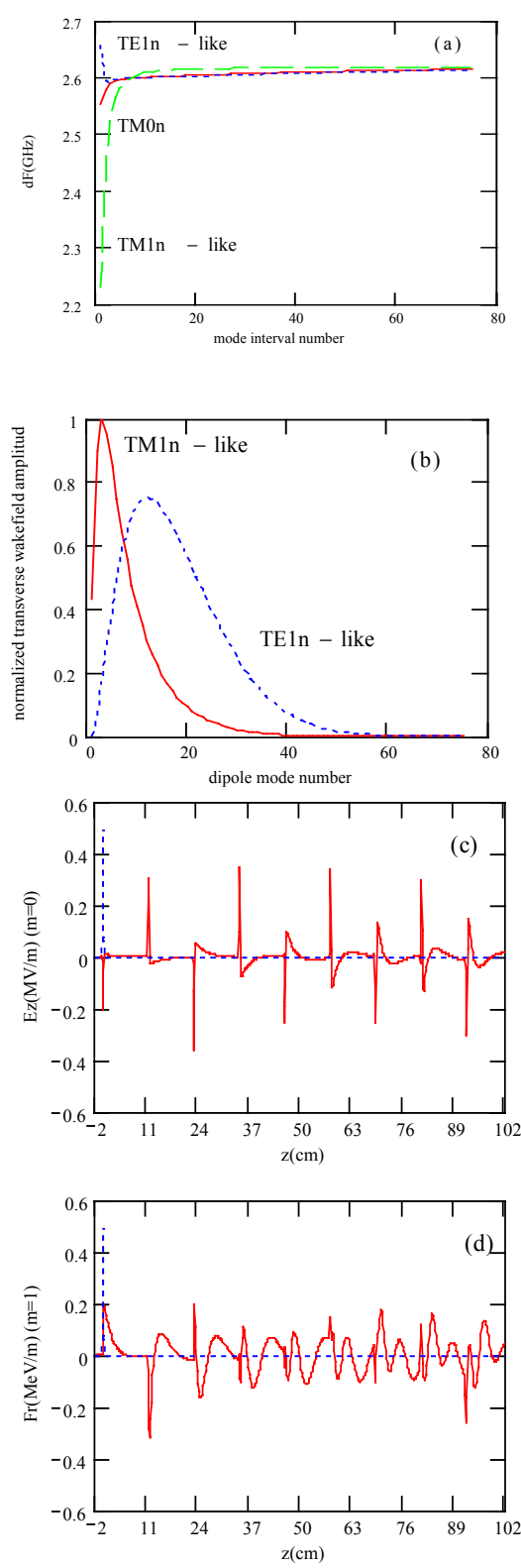

Fig.3 Calculated dielectric wakefield properties for TE dominated mode $(\mathrm{a}=5 \mathrm{~mm}, \mathrm{~b}=14.4 \mathrm{~mm}, \varepsilon=38.1$, $\sigma \mathrm{Z}=1 \mathrm{~mm}, \mathrm{Q}=1 \mathrm{nc}$ ) (a) frequency separation between adjacent modes, (b) normalized transverse wakefield amplitude, (c) longitudinal wakefield from a single bunch, (d) transverse force from a single bunch 